\title{
Reinstatement of Phascolosoma (Phascolosoma) varians Keferstein, 1865 (Sipuncula: Phascolosomatidae) based on morphological and molecular data
}

\author{
Itzahi Silva-Morales ${ }^{\text {Corresp. } 1}$ \\ ${ }^{1}$ Departamento de Sistemática y Ecología Acuática, El Colegio de la Frontera Sur, Chetumal, Quintana Roo, Mexico \\ Corresponding Author: Itzahi Silva-Morales \\ Email address: itzahi.silva@estudianteposgrado.ecosur.mx
}

Phascolosoma $(P$.$) varians, a sipunculan species known from the Greater Caribbean, was$ designated as a synonym of Phascolosoma $(P$.$) nigrescens, which was originally described$ from Fiji. Their synonymy was primarily based upon an interpretation that these two species were morphologically indistinguishable. After its designation as a synonym, no further detailed analyses of morphological or molecular characteristics were performed to corroborate the assumed widespread distribution of Phascolosoma $(P$.$) nigrescens. In this$ study, Phascolosoma $(P$.$) varians is redescribed, and notable differences between this$ species and its proposed senior synonym are presented. These two species differ in the shape of their hooks, the spatial attachment of nephridia to the body wall, and the morphology of the contractile vessel. Additionally, there is high genetic divergence between nucleotide sequences within their respective cytochrome c oxidase subunit 1 $(\mathrm{COI})$ genes, which supports the morphological data. Herein, the synonymy of Phascolosoma $(P$.$) varians with Phascolosoma (P$.$) nigrescens is rejected due to$ morphological and molecular differences. Furthermore, the assumed widespread distribution of Phascolosoma $(P$.$) nigrescens is still considered as questionable.$ 
2

3

4

5

6

8

9

10

10

11

12

13

14

15

16

17

18

19

20

21

22

23

24

25

26

27

28

29

30

31

32

33

34

35

36

37

38

39

40

\section{Reinstatement of Phascolosoma (Phascolosoma) varians Keferstein, 1865 (Sipuncula:} Phascolosomatidae) based on morphological and molecular data

Itzahí Silva-Morales $^{1}$

${ }^{1}$ Departamento de Sistemática y Ecología Acuática, El Colegio de la Frontera Sur, Chetumal, Quintana Roo

Corresponding Author:

Itzahí Silva-Morales ${ }^{1}$

${ }^{1}$ Av. Centenario, Chetumal, Quintana Roo, 77014, Mexico

Email address: itzahi.silva@estudianteposgrado.ecosur.mx

(3)




\section{Abstract}

42 Phascolosoma (P.) varians, a sipunculan species known from the Greater Caribbean, was

43 designated as a synonym of Phascolosoma $(P$.) nigrescens, which was originally described from

44 Fiji. Their synonymy was primarily based upon an interpretation that these two species were

45 morphologically indistinguishable. After its designation as a synonym, no further detailed

46 analyses of morphological or molecular characteristics were performed to corroborate the

47 assumed widespread distribution of Phascolosoma (P.) nigrescens. In this study, Phascolosoma

48 ( $P$.$) varians is redescribed, and notable differences between this species and its proposed senior$

49 synonym are presented. These two species differ in the shape of their hooks, the spatial

50 attachment of nephridia to the body wall, and the morphology of the contractile vessel.

51 Additionally, there is a high genetic divergence between nucleotide sequences within their

52 respective cytochrome $\mathrm{c}$ oxidase subunit $1(\mathrm{COI})$ genes, which supports the morphological data.

53 Herein, the synonymy of Phascolosoma $(P$.) varians with Phascolosoma $(P$.$) nigrescens is$

54 rejected due to morphological and molecular differences. Furthermore, the assumed widespread

55 distribution of Phascolosoma $(P$.) nigrescens is still considered as questionable.

56

\section{Introduction}

58

59

60

61

62

63

64

65

66

67

68

69

70

71

72

73

74

75

76

77

78

79

80

The phylum Sipuncula comprised of 320 species as recorded by Stephen \& Edmonds (1972), but after numerous revisions over a period of approximately twenty years by Edward and Norma Cutler, the total number was reduced to 149 valid species (Cutler, 1994). The reduced number of sipunculan taxa may call into question previous records, lead to possible taxonomic errors among field investigators, and suggest that most, if not all, species have been accounted for. However, after 1994, 13 new species have been described (Kawauchi \& Rice, 2009; Hylleberg, 2013; Saiz et al., 2015; Silva-Morales et al., 2019). Importantly, in the Greater Caribbean region alone, 38 valid species have been recorded, of which $40 \%$ correspond to species with type localities outside of the Greater Caribbean, with 7 of those species belonging to Phascolosomatidae (Quiroz-Ruiz \& Londoño-Mesa, 2015).

Reduction in the number of species by Cutler (1994) was performed by proposing extensive lists of synonyms, and when utilized by subsequent investigators, those lists have most likely led to a number of incorrect species identifications. For example, in the Greater Caribbean, P. (P.) nigrescens has been reported by Cutler \& Schulze (2004) from Barbados, Schulze \& Rice (2004) from Belize, Schulze (2005) from Panama, and Frontana-Uribe et al. (2018) from the Mexican Caribbean. These records may reflect the fact that Cutler (1994) considered the distribution of $P$. $\left(P\right.$.) nigrescens as widespread and circumtropical, found generally between $30^{\circ}$ $\mathrm{N}$ and $30^{\circ} \mathrm{S}$ in shallow waters of the Indian, Pacific, and Atlantic oceans.

The extensive synonymization approach by Cutler was proposed in part by an assumption that many sipunculan species have wide geographic distributions. Those with wide distributions were thought to be possible due to the high dispersal capability of species with teleplanic pelagosphera larvae, which were inferred to remain in the water column for up to six months base upon laboratory experiments (Rice, 1976). Phascolosoma (Phascolosoma) varians has a 
81 Category 4 developmental pattern (Rice, 1970): Indirect development with two pelagic larval 82 stages, trochophore and planktotrophic pelagosphera. Planktotrophic pelagosphera larvae can be 83 either short-lived (weeks) forms or larger long-lived (months) teleplanic larvae, as in $P$. (P.) 84 varians (Boyle \& Rice, 2014). The teleplanic larvae of the Phascolosomatidae are characterized 85 by having cuticular papillae (Scheltema \& Rice 1990).

$86 \quad$ However, recent molecular analyses revealed potential taxonomic problems at the species 87 level, where small morphological differences also were shown to correspond with distinct 88 species, thus rejecting previously assumed wide distributions for some of those species (Staton \& 89 Rice, 1999; Kawauchi \& Giribet, 2010; Schulze et al., 2012; Kawauchi \& Giribet, 2014; Johnson 90 91 92 93 et al., 2016; Silva-Morales et al., 2019). Because of this, Kawauchi, Sharma \& Giribet (2012) proposed to clarify the taxonomic status of each species through a meticulous case-by-case analysis, integrating molecular data while considering that some species names that are currently hidden under synonyms deserve to be restored. In regards to molecular data, the mitochondrial cytochrome oxidase c subunit I (COI) an efficient identification tool for metazoan species, making it the core fragment for DNA barcoding (Hebert, Ratnasingham \& de Waard, 2003).

Keferstein (1865) described Phascolosoma (Phascolosoma) varians from St. Thomas, West Indies, and $P$. (P.) nigrescens from Fiji. Cutler \& Cutler $(1983 ; 1990)$ reviewed the subgenus Phascolosoma (Phascolosoma), and redesignated 13 species, previously described and recorded from all around the world, as synonyms of $P$. $(P$.) nigrescens. The identification key by Cutler (1994) indicates that the diagnostic characters of $P$. $(P$.) nigrescens include the following: a distinct clear streak in particular hooks with observable swelling in the middle of vertical and horizontal portions. Phascolosoma $(P$.) varians was one of multiple species included as a junior synonym of $P$. (P.) nigrescens.

Herein, a detailed redescription of $P$. $(P$.) varians based upon topotypic specimens and additional material from other Caribbean localities is provided. Furthermore, $P$. $(P$.$) varians is$ reinstated due to both morphological and molecular differences that distinguish it from its proposed senior synonym, $P$. (P.) nigrescens.

\section{Materials \& Methods}

Specimens from the collections of the Marine Invertebrate Museum (UMML), Rosenstiel School of Marine and Atmospheric Science, University of Miami; Invertebrate Collections of the Florida Museum of Natural History (UF), University of Florida; and the Reference Collection of Benthos (ECOSUR) of El Colegio de la Frontera Sur, Chetumal, Mexico were reviewed.

Redescription of the species was primarily based upon a topotypic specimen, but additional materials from other Caribbean localities were also assessed for species-specific variations. Standardized descriptions included external and internal anatomy. The descriptions of hooks and papillae followed the terminology proposed by Cutler (1994). To measure the angle between the primary tooth and the hook, a line $X$ was drawn perpendicular to the base through the most anterior part of the concave side, and a line $Y$ was drawn from the tip until intersect $X$ in the middle of the point (see Cutler 1994:161-162, fig. 44A). 
121

122

123

124

125

126

127

128

129

130

131

132

133

134

135

136

137

138

139

140

141

142

143

144

145

146

147

148

149

150

151

152

153

154

155

156

157

158

159

160

Hooks and papillae were extracted with fine forceps for examination under an Olympus CH30 compound light microscope. Hooks were excised from three different regions (proximal, median and distal) of the ringed area of the introvert. Papillae were described from three different regions (anterior, median and posterior) of the trunk, and also from the distal introvert. Furthermore, these structures were examined using SEM to achieve a more detailed examination. For SEM preparation, the complete introvert was dehydrated through a series of increasing concentrations of hexamethyldisilazane (HMDS). Once air-dried, the introvert was mounted on an aluminum stub and coated with gold for observation with a JEOL JSM-6010Plus-LA scanning electron microscope at the Scanning Electron Microscopy Laboratory (LMEB), ECOSUR-Chetumal. Digital photographs of selected internal and external features were obtained with a Canon X6 digital camera mounted on a Leica MZ75 dissecting stereomicroscope. All images were rendered from a series of optical focal planes with HeliconFocus v6.7.1

(HeliconSoft 2007) to improve the depth of field for each specimen or set of specimens that were photographed.

For molecular analyses, eight COI sequences with an alignment length of $541 \mathrm{bp}$, from specimens identified as Phascolosoma $(P$.) nigrescens were retrieved from GenBank. One of them from Barbados (DQ300139), two from Florida (DQ300142, AY161122), one from Broome, Australia (DQ300143), two from New Caledonia (JN865121, JN865122), one from Israel (DQ300140), and another from South Africa (DQ300141). Also, a COI sequence from Phascolosoma (P.) granulatum Leuckart, 1828 (DQ300138) and two sequences from Phascolosoma (P.) agassizii Keferstein, 1866 (JQ904338, JQ904337) were included for comparison.

All sequences were aligned using the ClustalW method. Selection of the best model of substitution was determined according to the lowest Bayesian Information Criterion scores (BIC). From the BIC results, the Tamura 3-parameter (Tamura 1992) model with a discrete Gamma distribution $(+\mathrm{G})$ with five categories, assuming a fraction of sites is evolutionarily invariable $(+\mathrm{I})$, was selected to construct a tree by maximum likelihood analysis. The Kimura 2parameter model (Kimura 1980) was used to estimate the average evolutionary divergence over sequence pairs within and between species. All analyses were carried out with Mega 7 (Kumar, Stecher \& Tamura, 2015).

\section{Results}

\section{Systematics}

Family Phascolosomatidae Stephen \& Edmonds, 1972

Phascolosoma Leuckart, 1828

Phascolosoma (Phascolosoma) Leuckart, 1828 
161

162

163

164

165

166

167

168

169

170

171

172

173

174

175

176

177

178

179

180

181

182

183

184

185

186

187

188

189

190

191

192

193

194

195

196

197

198

199

200

Type species. Phascolosoma granulatum Leuckart, 1828

Diagnosis. Body wall muscles separated into distinct bands. Spindle muscle attached posteriorly; introvert hooks without accessory spinelets (after Cutler 1994).

\section{Phascolosoma (Phascolosoma) varians Keferstein, 1865 reinstated}

Phascolosoma varians Keferstein 1865: 424-426, pl. 32, Fig. 22. de Quatrefages 1865: 623.

Wesenberg-Lund 1954: 7-8. Rice \& Macintyre 1982: 314.

Phymosoma varians: Selenka 1883: 69-70, pl. 9, figs. 124-127. Shipley 1890: 1-24, fig. pls. 1-4, figs. 1-32.

Physcosoma varians ten Broeke 1925: 85.

Phascolosoma (Phascolosoma) varians: Stephen \& Edmonds 1972: 327-328, fig. 39I.

Phascolosoma nigrescens Cutler 1994 (partim): 179-181; Cutler \& Schulze 2004: 226; Schulze 2005: 526; Frontana-Uribe et al. 2018:174, fig. 5a-b; (non Keferstein, 1865).

\section{Material examined}

USA, Florida. UMML 26.5, 2 specimens, Bear Cut, Key Biscayne, 2543'54.33"N, 8009'26.16"W, May 7, 1961, coll. P. Robertson. UMML s/n, 1 specimen. Margot Fish Shoal, Dade Co, Apr 5, 1966, coll. G. Hendrix, bored in coral rubble. UF 292, 1 specimen, N of St. Petersburg, 28²7'33.12"N, 84¹6'18.48"W, 30 m, Mar 13, 2011, colls. G. Paulay, N. Evans, F. Michonneau, C. Thacker, R. Williams, A. Baeza. UF 293, 1 specimen, N of St. Petersburg, $28^{\circ} 27^{\prime} 33.12^{\prime \prime} \mathrm{N}, 84^{\circ} 16^{\prime} 18.48^{\prime \prime} \mathrm{W}$, hard ground with sponges, $30 \mathrm{~m}$, Mar 13, 2011, coll. G. Paulay.

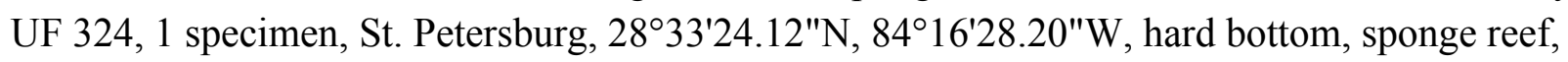
27 m, May 24, 2012, coll. J. Slapcinsky. UF 325, 1 specimen, St. Petersburg, 28³9'03.96"N, 84²3'03.84"W, 26-30 m, hard bottom, sponge reef, May 25, 2012, colls. G. Paulay, N. Evans, F. Michonneau. Mexico, Mexican Caribbean, Isla Contoy. ECOSUR-S62, 3 specimens, Morro Norte, 2128'32.79"N, 86²7'30.13"W, coralline rock, 2.5 m, Feb 26, 2008, colls. S. SalazarVallejo, L. Carrera-Parra. ECOSUR-S63, 2 specimens. Punta Sur, 2127'37.10"N, $86^{\circ} 47^{\prime} 04.60^{\prime \prime} \mathrm{W}$, coralline rock, $1.5 \mathrm{~m}$, Mar 2, 2001, colls. S. Salazar-Vallejo, L. Carrera-Parra. ECOSUR-S69, 1 specimen, Punta Sur, 21 ${ }^{\circ} 7^{\prime} 37.10^{\prime \prime} \mathrm{N}, 86^{\circ} 47^{\prime} 04.60^{\prime \prime} \mathrm{W}$, coralline rock, $1.5 \mathrm{~m}$, Feb 28, 2001, colls. S. Salazar-Vallejo, L. Carrera-Parra. ECOSUR-S70, 1 specimen, Ixlache reef, $21^{\circ} 26^{\prime} 02.52^{\prime \prime N}, 86^{\circ} 46^{\prime} 56.16^{\prime \prime W}$, coralline rock, 2 m, Feb 25, 2008, colls. S. Salazar-Vallejo, L. Carrera-Parra. Cancun, Punta Nizuc. ECOSUR-S87, 1 specimen, 21 ${ }^{\circ} 01^{\prime} 41.92^{\prime \prime N}$, 8646'45.72"W, coralline rock, 2.6 m, Aug 31, 1997, colls. S. Salazar-Vallejo, L. Carrera-Parra, M. Ruiz-Zárate. ECOSUR-S88, 2 specimens, $21^{\circ} 01^{\prime} 17.06^{\prime \prime} \mathrm{N}, 86^{\circ} 46^{\prime} 45.95^{\prime \prime} \mathrm{W}$, coralline rock, 4 m, Sep 1, 1997, colls. S. Salazar-Vallejo, L. Carrera-Parra, M. Ruiz-Zárate. ECOSUR-S89, 3 specimens, $21^{\circ} 01^{\prime} 17.06^{\prime \prime N}, 86^{\circ} 46^{\prime} 45.95^{\prime \prime}$, coralline rock, 4 m, Sep 1, 1997, colls. S. Salazar- 
201 Vallejo, L. Carrera-Parra, M. Ruiz-Zárate. ECOSUR-S90, 2 specimens, $21^{\circ} 01^{\prime} 17.06 " \mathrm{~N}$, $20286^{\circ} 46^{\prime} 45.95^{\prime \prime W}$, coralline rock, 4 m, Sep 1, 1997, colls. S. Salazar-Vallejo, L. Carrera-Parra, M. 203 Ruiz-Zárate. Playa del Carmen. ECOSUR-S86, 4 specimens, Navega pier, 20³7'12.07"N, 204 8704'26.63"W, fouling, 1 m, Aug 23, 2003, coll. M. Tovar-Hernández. Cozumel. ECOSUR205 S64, 14 specimens, Playa Azul, 20³2'51.98"N, 8655'46.45"W, coralline rock, $1 \mathrm{~m}$, Mar 25, 206 2001, colls. S. Salazar-Vallejo, L. Carrera-Parra. ECOSUR-S65, 1 specimen, in front to 207 SEDENA, 20³1'00.61"N, 8656'45.52"W, coralline rock, $1.5 \mathrm{~m}$, Mar 24, 2001, colls. S. 208 Salazar-Vallejo, M. Londoño-Mesa. Tulum. ECOSUR-S66, 3 specimens, Playa Aventuras, $20920^{\circ} 21^{\prime} 47.20^{\prime \prime} \mathrm{N}, 8^{\circ} 19^{\prime} 53.10^{\prime \prime} \mathrm{W}$, coralline rock, 1.5 m, Feb 28, 1999, colls. S. Salazar-Vallejo, J. 210 Bastida-Zavala. ECOSUR-S91, 1 specimen, Playa Aventuras, 20²1'47.20"N, 87¹9'53.10"W, 211 coralline rock, 1.5 m, Feb 18, 2001, colls. S. Salazar-Vallejo, L. Carrera-Parra. ECOSUR-S92, 4 212 specimens, Ana y José beach, 2009'24.22"N, 87²7'13.74"W, coralline rock, $1 \mathrm{~m}$, Feb 11, 2001, 213 colls. S. Salazar-Vallejo, J. Bastida-Zavala, J. Ruiz-Ramírez. ECOSUR-S96, 3 specimens, Punta 214 Piedra, 20¹0'38.94"N, 87²6'42.28"W, coralline rock, 1.5 m, Feb 11, 2001, colls. S. Salazar215 Vallejo, L. Carrera-Parra, M. Tovar-Hernández. Mahahual. ECOSUR-S67, 1 specimen, $25 \mathrm{~m}$ 216 off coast, $18^{\circ} 43^{\prime} 27.09^{\prime \prime} \mathrm{N}, 87^{\circ} 42^{\prime} 3.64^{\prime \prime} \mathrm{W}$, reef lagoon, rocky substrate with sediment, $0.75 \mathrm{~m}$, 217 Oct 1, 1996, colls. S. Salazar-Vallejo, L. Carrera-Parra. ECOSUR-S71, 1 specimen, in sponge, 218 Jul 21, 1998. ECOSUR-S72, 2 specimens, 50 m off coast, 1843'38.68"N, 8741'56.81"W, 219 coralline rock, 2 m, Mar 4, 1998, colls. S. Salazar-Vallejo, L. Carrera-Parra. ECOSUR-S73, 6

220

221

222

223

224

225

226

227

228

229

230

231

232

233

234

235

236

237

238

239

240 specimens, reef lagoon near to back reef, $18^{\circ} 42^{\prime} 34.01^{\prime \prime N}, 8^{\circ} 42^{\prime} 31.22^{\prime \prime} \mathrm{W}$, coralline rock, $1.5 \mathrm{~m}$, Jan 9, 2001, colls. P. Salazar-Silva, J. Bastida-Zavala, M. Tovar-Hernández, S. Salazar-Vallejo, L. Carrera-Parra. ECOSUR-S74, 1 specimen, fore reef, $18^{\circ} 42^{\prime} 43.32^{\prime \prime N}$, 87² $42^{\prime} 22.51^{\prime \prime} \mathrm{W}$, coralline rock, 15 m, Jun 6, 1998, coll. M. Ruiz-Zárate. ECOSUR-S75, 2 specimens, reef lagoon, $18^{\circ} 42^{\prime} 36.23^{\prime \prime N}, 87^{\circ} 42^{\prime} 31.60^{\prime \prime W}$, coralline rock, $1.5 \mathrm{~m}$, Dec 1, 2000, colls. S. Salazar-Vallejo, L. Carrera-Parra. ECOSUR-S76, 2 specimens, reef crest, $18^{\circ} 43^{\prime} 06.17^{\prime \prime} \mathrm{N}, 87^{\circ} 42^{\prime} 14.17^{\prime \prime} \mathrm{W}$, coralline rock, $1 \mathrm{~m}$, Jul 21, 1998, colls. S. Salazar-Vallejo, L. Carrera-Parra. ECOSUR-S77, 5 specimens, back reef, $18^{\circ} 42^{\prime} 31.30^{\prime \prime} \mathrm{N}, 87^{\circ} 42^{\prime} 30.39^{\prime \prime} \mathrm{W}$, coralline rock, $2 \mathrm{~m}$, Mar 22, 2000, colls. S. SalazarVallejo, L. Carrera-Parra. ECOSUR-S78, 1 specimen, reef lagoon, $18^{\circ} 42^{\prime} 36.17^{\prime \prime N}$, $87^{\circ} 42^{\prime} 32.65^{\prime \prime} \mathrm{W}$, coralline rock, $1.5 \mathrm{~m}$, Mar 21, 2000, colls. J. Bastida-Zavala, P. Salazar-Silva. ECOSUR-S79, 5 specimens, old wooden pier, $18^{\circ} 42^{\prime} 41.95^{\prime \prime} \mathrm{N}, 8^{\circ} 42^{\prime} 35.98^{\prime \prime} \mathrm{W}$, fouling, $1 \mathrm{~m}$, Feb 24, 2001, colls. P. Salazar-Silva, J. Bastida-Zavala, M. Tovar-Hernández, S. Salazar-Vallejo, L. Carrera-Parra, L. Harris. ECOSUR-S80, 8 specimens, old wooden pier, $18^{\circ} 42^{\prime} 41.95^{\prime \prime} \mathrm{N}$, $87^{\circ} 42^{\prime} 35.98^{\prime \prime W}$, fouling, $1 \mathrm{~m}$, Mar 18, 2001, colls. P. Salazar-Silva, J. Bastida-Zavala, M. TovarHernández, S. Salazar-Vallejo, L. Carrera-Parra. ECOSUR-S81, 14 specimens, reef lagoon, $18^{\circ} 43^{\prime} 22.73^{\prime \prime N}, 87^{\circ} 42^{\prime} 03.08^{\prime \prime} \mathrm{W}$, coralline rock, $1.5 \mathrm{~m}$, Mar 28, 2001, colls. L. Carrera-Parra, M. Londoño-Mesa, S. Salazar-Vallejo. ECOSUR-S82, 1 specimen, reef lagoon, $18^{\circ} 43^{\prime 25.14} \mathrm{~N}$, $87^{\circ} 42^{\prime} 01.75^{\prime \prime} \mathrm{W}$, coralline rock, 1.5, Jan 10, 2001, colls. L. Carrera-Parra, M. Londoño-Mesa, S. Salazar-Vallejo. ECOSUR-S83, 12 specimens, reef lagoon, $18^{\circ} 43^{\prime} 24.93^{\prime \prime N}, 87^{\circ} 42^{\prime} 02.95^{\prime \prime} \mathrm{W}$, coralline rock, 1 m, Jan 19, 2001, colls. P. Salazar-Silva, J. Bastida-Zavala, M. TovarHernández, S. Salazar-Vallejo, L. Carrera-Parra. ECOSUR-S84, 2 specimens, reef lagoon, 
$241 \quad 18^{\circ} 43^{\prime} 24.93^{\prime \prime N}, 87^{\circ} 42^{\prime} 02.95^{\prime \prime} \mathrm{W}$, coralline rock, $1 \mathrm{~m}$, Nov 30, 2000, colls. S. Salazar-Vallejo, L. 242 Carrera-Parra. ECOSUR-S85, 13 specimens, reef lagoon, 1843'21.01"N, 87² $2^{\prime} 04.28^{\prime \prime} \mathrm{W}$, 243 coralline rock, 1.5 m, Feb 24, 2001, colls. P. Salazar-Silva, J. Bastida-Zavala, M. Tovar244 Hernández, S. Salazar-Vallejo, L. Carrera-Parra, L. Harris. ECOSUR-S93, 1 specimen, punta 245 Rio Bermejo, 1841'07.53"N, 8743'05.83"W, corralline rock, $1 \mathrm{~m}$, May 17 2002, colls. S. 246 Salazar-Vallejo, M. García-Madrigal. ECOSUR-S94, 16 specimens, punta Rio Indio, $247 \quad 18^{\circ} 48^{\prime} 29.63^{\prime \prime} \mathrm{N}, 87^{\circ} 39^{\prime} 58.82^{\prime \prime} \mathrm{W}$, coralline rock, $1.7 \mathrm{~m}$, Mar 17, 2001, coll. L. Carrera-Parra. 248 Xahuayxol, ECOSUR-S58, 8 specimens, reef lagoon, 18³0'11"N, 8745'29"W, coralline rock, 2

249

250

251

252

253

254

255

256

257

258

259

260

261

262

263

264

265

266

267

268

269

270

271

272

273

274

275

276

277

278

279

280 m, Jun 1 1997, colls. S. Salazar-Vallejo, L. Carrera-Parra. ECOSUR-S59, 1 specimen, back reef, 18³0'11.45"N, 8745'21.46"W, coralline rock, 12 m, Oct 30 1997, colls. R. Saenz-Morales, S. Salazar-Vallejo, L. Carrera-Parra. ECOSUR-S60, 4 specimens, reef lagoon, $120 \mathrm{~m}$ off coast, $18^{\circ} 30^{\prime} 41.34^{\prime \prime N}, 8^{\circ} 45^{\prime} 24.63^{\prime \prime W}$, coralline rock, 1.5 m, Oct 31 1997, colls. S. Salazar-Vallejo, L. Carrera-Parra. ECOSUR-S61, 11 specimens, reef lagoon, 18³0'39.77"N, 87²5'24.80"W, coralline rock, 1.8 m, Jun 4, 1998, colls. S. Salazar-Vallejo, L. Carrera-Parra. ECOSUR-S68, 3 specimens, reef lagoon, 18³0'39.04"N, 8745'25.09"W, coralline rock, 1.7 m, Sep 27, 1996, colls. S. Salazar-Vallejo, L. Carrera-Parra. ECOSUR-S95, 21 specimens, reef lagoon, $18^{\circ} 30^{\prime} 12.46^{\prime \prime N}, 87^{\circ} 45^{\prime} 29.79^{\prime \prime} \mathrm{W}$, coralline rock, 2 m, Jun 1, 1997, colls. S. Salazar-Vallejo, L. Carrera-Parra. ECOSUR-S97, 1 specimen, 100 m off coast, 18 30'41.43"N, 8745'25.16"W, coralline rock, 2 m, Sep 28, 1996, coll. S. Salazar-Vallejo, L. Carrera-Parra. ECOSUR-S98, 1 specimen, reef lagoon, 18³0'12.46"N, 8745'29.79"W, coralline rock, 2 m, Jun 1, 1997, colls. S. Salazar-Vallejo, L. Carrera-Parra. ECOSUR-S99, 6 specimens, reef lagoon, 18³0'13.71"N, 8745'31.50"W, coralline rock, 1 m, Jun 2 1998, colls. S. Salazar-Vallejo, L. Carrera-Parra. ECOSUR-S100, 2 specimens, 100 m off coast, 18 $30^{\prime} 15.08^{\prime \prime} \mathrm{N}, 87^{\circ} 45^{\prime} 30.98^{\prime \prime} \mathrm{W}$, in sediment with Thalassia testudinum, 2 m, Sep 27, 1996, colls. S. Salazar-Vallejo, L. Carrera-Parra. Xcalak. ECOSUR-S101, 9 specimens, back reef, 18¹5'50.40"N, 8749'31.12"W, coralline rock, $1.7 \mathrm{~m}$, Oct 25, 2002, colls. S. Salazar-Vallejo, L. Carrera-Parra, P. Salazar-Silva, M. Londoño-Mesa. Nicaragua, Nicaraguan Caribbean. UMML 000, 1 specimen, R/V Pillsbury, Cruise 7101, sta. $1338,12^{\circ} 52^{\prime} 00^{\prime \prime} \mathrm{N}, 82^{\circ} 35^{\prime} 17.98^{\prime \prime} \mathrm{W}$, hillocks or low rounded mounds, $28 \mathrm{~m}$, Jan 29, 1971, coll. G. Voss. Colombia, Providence Island. UMML 000, 1 specimen, R/V Pillsbury, Cruise 7101, sta. $1349,13^{\circ} 33^{\prime} 00^{\prime \prime} \mathrm{N}, 81^{\circ} 28^{\prime} 00^{\prime \prime} \mathrm{W}$, patch reef slightly southwest of Low Island surrounded by sand bottom, 3 m, Jan 30, 1971. Dominican Republic. UMML 000, 2 specimens. R/V Pillsbury, Cruise 7006, sta. 1272, off Cabo Rojo, 1752'41.98"N, 7141'12.01"W, 20-27 m, Jul 17, 1970, coll. J. Staiger. Turks and Caicos. UMML 000. 1 specimen, Pillsbury Cruise 7106, sta. 1423,

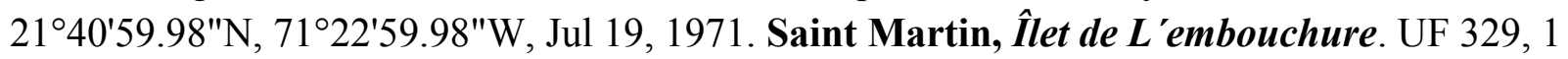
specimen, $18^{\circ} 04^{\prime} 1.2^{\prime \prime} \mathrm{N}, 63^{\circ} 00^{\prime} 39.6^{\prime \prime} \mathrm{W}$, reef flat lagoon with seagrass, $1 \mathrm{~m}$, Apr 9, 2012, colls. G. Paulay, J. Slapcinsky, M. Bemis. UF 330, 5 specimens, $18^{\circ} 04^{\prime} 01.20^{\prime \prime} \mathrm{N}, 63^{\circ} 00^{\prime} 43.20^{\prime \prime} \mathrm{W}$, reef flat lagoon with seagrass, 0-1 m, Apr 9, 2012, colls. G. Paulay, J. Slapcinsky, M. Bemis. UF 338. 1 specimen, $18^{\circ} 04^{\prime} 01.20^{\prime \prime} \mathrm{N}, 63^{\circ} 00^{\prime} 43.20^{\prime \prime} \mathrm{W}$, reef, $1 \mathrm{~m}$, Apr 17, 2012, coll. A. Anker. UF 340, 1 specimen, $18^{\circ} 04^{\prime} 01.20^{\prime \prime} \mathrm{N}, 63^{\circ} 00^{\prime} 43.20^{\prime \prime} \mathrm{W}$, reef, $1 \mathrm{~m}$, Apr 17, 2012, coll. A. Anker. UF 347, 1 specimen, $18^{\circ} 04^{\prime} 01.20^{\prime \prime} \mathrm{N}, 63^{\circ} 00^{\prime} 43.20^{\prime \prime} \mathrm{W}$, reef, 0-1 m, Apr 17, 2012, colls. G. Paulay, J. 
281 Slapcinsky, A. Anker. UF 359, 3 specimens, steep rubbly reef slope, 2-10 m, Apr 22, 2012, colls.

282 G. Paulay, F. Michonneau. Mont Vernon, UF 332, 1 specimen, Little Key, $18^{\circ} 06^{\prime} 00^{\prime \prime} \mathrm{N}$, $28363^{\circ} 01^{\prime} 19.20^{\prime \prime} \mathrm{W}$, seagrass, sand, rocks, 0-2 m, Apr 11, 2012, coll. J. Slapcinsky. Grande Caye,

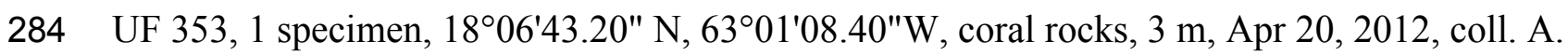
285 Anker. Rocher Créole. UF 352. 1 specimen, 1807'04.80"N, 6303'21.60"W, reef, 9 m, Apr 18, 286 2012, colls. G. Paulay, J. Slapcinsky, M. Bemis. NE side of St. Martin, UF 336, 1 specimen, $28718^{\circ} 07^{\prime} 48^{\prime \prime} \mathrm{N}, 63^{\circ} 00^{\prime} 18^{\prime \prime} \mathrm{W}$, canyon with sponges, in algae, $13 \mathrm{~m}$, Apr 11, 2012, coll. R. Renoux. 288 Île Tintammare, UF 361, 3 specimens, Chicot, windward side of the island, $18^{\circ} 06^{\prime} 7.2^{\prime \prime} \mathrm{N}$, $28962^{\circ} 58^{\prime} 58.80^{\prime \prime} \mathrm{W}$, reef, in rubble, 12-15 m, Apr 23, 2012, coll. G. Paulay. Caye Verte, UF 364, 1 290 specimen, $18^{\circ} 05^{\prime} 24^{\prime \prime} \mathrm{N}, 63^{\circ} 00^{\prime} 43.2^{\prime \prime} \mathrm{W}$, reef with sand and grass, $7 \mathrm{~m}$, Apr 25, 2012, coll. M. 291 Bemis. Panama. Bocas del Toro. UF 126, 1 specimen, coll. C. Meyer. UF 485. 1 specimen, 292 Punta Puebla, 9²2'01.20"N, 82¹7'27.60"W, May 16, 2016, colls. M. Leray, F. Michonneau, R. 293 Lasley. UF 486, 1 specimen, Punta Juan, 9¹8'03.60"N, 82¹7'38.40"W, May 16, 2016, coll. R. 294 Lasley. UF 494, 1 specimen, Runway, 9²0'31.20"N, 82¹5'36"W, May 23, 2016, colls. M.

295

296

297

298

299

300

301

302

303

304

305

306

307

308

309

310

311

312

313

314

315

316

317

318

319

320

Leray, F. Michonneau, R. Lasley. UF 499. 1 specimen, Marina, 9¹9'51.60"N, 82¹4'38.40"W, May 28, 2016, colls. M. Leray, F. Michonneau, R. Lasley. UF 500, 2 specimens, Marina, $9^{\circ} 19^{\prime} 51.60 " \mathrm{~N}, 82^{\circ} 14^{\prime} 38.40^{\prime \prime}$, May 28, 2016, colls. M. Leray, F. Michonneau, R. Lasley. UF 501, 1 specimen, Cayo Hermanas, $9^{\circ} 16^{\prime} 04.80^{\prime \prime} \mathrm{N}, 82^{\circ} 21^{\prime} 07.20^{\prime \prime} \mathrm{W}$, May 30, 2016, colls. M.

Leray, F. Michonneau, R. Lasley. UF 503, 1 specimen. Cayo Hermanas, 9¹6'04.80"N, $82^{\circ} 21^{\prime} 07.20^{\prime \prime W}$, May 31, 2016, colls. M. Leray, F. Michonneau, R. Lasley. UF 541, 1 specimen, Salt Creek, 9०16'48"N, 8206'07.20"W, outer reef, Agaricia reef, 4-4.5 m, May 22, 2016, colls. M. Leray, F. Michonneau, R. Lasley. UF 542, 1 specimen, Runway, 9²0'31.20"N, 82¹5'36"W, May 23, 2016, colls. M. Leray, F. Michonneau, R. Lasley, 4-4.5 m, lagoon fringing reef, Agaricia reef.

\section{Redescription}

Male specimen from St. Martin, West Indies (UF 332).

External anatomy. Trunk $14 \mathrm{~mm}$ in length (Fig. 1A); light brown with some darker patches; papillae dark brown and light brown distributed randomly, most of them on the dorsal region, scarce ventrally; papillae conglomerated in anal region (Fig. 1D) and caudal region (Fig. $1 \mathrm{~F}$ ), most dispersed in median region (Fig. 1E).

Introvert $10 \mathrm{~mm}$ length (Fig. 1A), dark brown, uniform color ventrally and dorsally, with ill-defined transversal bands light brown color (Fig. 1B), distal introvert with similar color pattern as trunk, extensible collar small. Introvert papillae dark brown, smaller than those of trunk (Fig. 1C), located between rings of hooks, starting from the first ring (Fig. 2H), these are likely secretory or sensory papillae. Nuchal organ with wavy contour.

Hooks laterally compressed, arranged in sixty complete rings and some incomplete rings, probably due to abrasion. Rings followed by a zone with scattered hooks. Proximal hooks (Fig. $2 \mathrm{~A}, \mathrm{~F}$ ) with an angle of $90^{\circ}$ between line $\mathrm{X}$ and $\mathrm{Y}$; length of distal tip never projecting beyond

Peer) reviewing PDF | (2020:08:51760:1:0:NEW 22 Sep 2020) 
321 the base of the hook; secondary tooth rounded; internal clear streak (apical canal) expanded near

322

323

324

325

326

327

328

329

330

331

332

333

334

335

336

337

338

339

340

341

342

343

344

345

346

347

348

349

350

351

352

353

354

355

356

357

358

359

360

to midpoint of vertical and middle horizontal portions of hook. Hooks of median region with a larger secondary tooth (Fig. 2B, G), distal hooks (Fig. 2C) with principal tooth smaller than its base, almost $25 \%$ less. Proximal hooks with external border bent squarely; hooks of the median region of the introvert with progressively rounder bent border; distal hooks with evenly rounded external border.

SEM revealed growth stages of hooks and papillae (Fig. $2 \mathrm{H}$ ); smaller in the proximal introvert and proximal trunk and larger at the distal introvert and distal trunk. Introvert papillae with three stages of development. First stage: the smallest, spherical with a ring of short apical protrusions (Fig. 2K) "dome shape" (fide Cutler 1994). Second stage: medium size, appearance of two units, the smallest with a ring of short apical protrusions, and a broad base (Fig. 2J) "mammillate form" (fide Cutler 1994). Third stage: largest, conical (Fig. 2I) "cone shape" (fide Cutler 1994).

Internal anatomy (Fig. 2D). A pair of nephridia occupying $80 \%$ of trunk length, open at the same level as anus. Longitudinal musculature divided into 23 individual and anastomosed bands in the median trunk. Two pairs of retractor muscles; ventral pair attached to 8 longitudinal bands starting from the third band after ventral nerve cord (Fig. 2E), dorsal pair attached to 5 longitudinal bands starting from the fifth band after ventral nerve cord. Contractile vessel without swelling or villi. Spindle muscle attached posteriorly.

Habitat. In coralline rock and hard bottom, 1 to $30 \mathrm{~m}$ depth.

Distribution. Greater Caribbean from Florida to Venezuela.

Remarks. Keferstein (1865) described Phascolosoma (Phascolosoma) varians from St. Thomas, West Indies and $P$. $(P$.) nigrescens from Fiji. Although Keferstein's descriptions were well illustrated, Selenka (1883) produced an improved set of drawings from the type materials (Fig. 3). Keferstein (1865) recognized each species as follows: Phascolosoma varians with a body three to four times as long as thick; introvert as long or longer than the body; closely spaced rows of hooks, highly variable in number (12-90), which often only cover the anteriormost part of the trunk; hooks very broad, with an upper right-angled tip ( $0.072 \mathrm{~mm}$ high, $0.092 \mathrm{~mm}$ wide); with 20-28 short tentacles, standing in two rows at the side; longitudinal muscles about thirty, but in many cases anastomosed as longitudinal strands; contractile vessel simple, without lateral sags; nephridia very long, attached in the anterior third by a mesentery. Phascolosoma nigrescens has a trunk about four times as long as thick; introvert longer than trunk; numerous hooks forming rings situated very close to each other in the anterior end of the trunk; hooks flattened with an lower right-angled tip ( $0.084 \mathrm{~mm}$ high, $0.084 \mathrm{~mm}$ wide); over twenty tentacles in several rows; muscles separated in about 24 longitudinal strands with few anastomosed bands; contractile vessel on the esophagus with many small, lateral sags; nephridia attached along almost their entire length by a wide mesentery.

Peer) reviewing PDF | (2020:08:51760:1:0:NEW 22 Sep 2020) 
361

362

363

364

365

366

367

368

369

370

371

372

373

374

375

376

377

378

379

380

381

382

383

384

385

386

387

388

389

390

391

392

393

394

395

396

397

398

399

400

Cutler \& Cutler (1990) reviewed the subgenus Phascolosoma (Phascolosoma), and designated $P$. (P.) varians, together with 9 other species from different regions of the world, as junior synonyms of $P$. (P.) nigrescens. At that time, Cutler \& Cutler (1990) suggested there were "no consistent differences" between these two species. Their decision appears to be primarily based upon variation associated with hook morphology. According to Cutler \& Cutler (1990): "One possible hypothesis is that hook morphology is determined by more than one pair of genes and that allelic frequencies vary from place to place. The alleles for sharp angle and large secondary tooth occur at a high frequency in the Caribbean and a low frequency in the IndoWest Pacific. . . P. varians is the junior name because it was described later on the page". No molecular evidence was ever provided in support of the allelic hypothesis, or any other genetic differences supporting proposed junior synonyms. Subsequently, Cutler (1994) published his synonymy upon further consideration of the morphological differences between $P$. $(P$.) nigrescens and $P$. (P.) varians.

Herein, reexamination and redescription of $P$. $(P$.$) varians, revealed clear morphological$ differences concerning its previously designated senior synonym. The most important features that distinguish these two species include the shape of the hooks, the attachment of nephridia to the body wall, and morphology the contractile vessel. Phascolosoma (P.) varians has hooks with a rounded secondary tooth; the base of the hook is broader than high; most of the anterior hooks (Fig. 2A and $2 \mathrm{~F}$ ) possess a distal tip at a $90^{\circ}$ angle with respect to the perpendicular axial line of the hook; the contractile vessel is simple; nephridia are attached to body wall in the anterior third. $P(P$.) nigrescens has hooks with a square secondary tooth; the base of hook is as broad as high; most of the anterior hooks possess a distal tip with less than a $90^{\circ}$ angle with respect to the perpendicular axial line of the hook; a contractile vessel on the esophagus with many small, lateral sags; nephridia are attached almost along their entire length.

The wavy contour of the nuchal organ and specific attachments of the retractor muscles were not described for both species by Keferstein in 1865, nevertheless, these features are now described. Keferstein (1865) refers to "tentacles in two rows or many rows", which may explain the wavy contour of the nuchal organs I observed. The differences between the number of tentacles is not useful for separating these species because they appear to vary with the development stage of the specimen, and that variation overlaps between these species. Because of the difficulty of establishing the exact number of longitudinal muscle bands, this characteristic should be considered cautiously. Additionally, the number of rings of hooks is variable between these species, and the loss of rings is not uncommon. Papillae are also inappropriate characters for distinguishing these species as their differences are minor across most of the body surface.

Phascolosoma (Phascolosoma) granulatum was included for comparison in the molecular analyses. The species can be easily distinguished from $P$. $(P$.) varians for having hooks with a basal triangle and lacking bands of pigmentation in the introvert, while $P$. $(P$.) varians lacks a basal triangle in the hooks and the bands of pigmentation are conspicuous.

\section{Molecular analyses}

Peer) reviewing PDF | (2020:08:51760:1:0:NEW 22 Sep 2020) 
401

402

403

404

405

406

407

408

409

410

411

412

413

414

415

416

417

418

419

420

421

422

423

424

425

426

427

428

429

430

431

432

433

434

435

436

437

438

439

440

Herein, the synonymy of Phascolosoma $(P$.) varians with Phascolosoma $(P$.) nigrescens is rejected due to morphological differences. Additionally, there is a high genetic divergence between nucleotide sequences within their respective cytochrome c oxidase subunit 1 (COI) genes, which supports the morphological data.

The first three sequences (Fig. 4) (top-down) correspond to Phascolosoma (P.) varians from the Greater Caribbean. This species had been determined as Phascolosoma (P.) nigrescens by past authors and registered as such it in GenBank. These sequences were grouped with a low intraspecific variation of $2.6 \%$. The localities of those sequences are Florida and Barbados; preserved specimens from the same localities were revised morphologically to support the correct identification of the species.

The two sequences of Phascolosoma $(P$.$) nigrescens from New Caledonia constitute a$ group clearly separated from Phascolosoma $(P$.) varians from the Greater Caribbean (genetic mean distance $24 \%$ ). There are two crucial facts to consider. First: New Caledonia is the closest locality to Fiji, the type locality of $P$. $(P$.) nigrescens. The high genetic divergences between the specimens from New Caledonia and those from the Greater Caribbean supports the morphological differences between both species and reinforce the reinstatement of $P$. $(P$.) varians. Second: the intraspecific variation between both sequences is very high (18\%), almost the same value of the interspecific variation of the other groups in this analysis. This value suggests that it is highly likely that these two sequences represent different morphotypes, and either one of them would correspond with Phascolosoma $(P$.$) nigrescens.$

The values of the genetic distance between Phascolosoma $(P$.$) nigrescens from Israel,$ South Africa and, Broome, Australia, varies from 18 to $26 \%$ regarding Phascolosoma (Phascolosoma) varians from the Greater Caribbean. These values are similar to the results of Silva-Morales et al. (2019), where they found a genetic distance of $19 \%$ between Antillesoma antillarum (Greater Caribbean) and A. mexicanum (Southern Mexican Pacific). The present analysis reveals that Phascolosoma (Phascolosoma) nigrescens is a species complex resulting from the incipient morphological analysis. This study shows that Phascolosoma (P.) varians from the Greater Caribbean is well differentiating morphologically and genetically of Phascolosoma (P.) nigrescens; however, a detailed morphological revision of this species complex is needed.

\section{Discussion}

The genetic analysis suggests the following considerations: 1) the specimens from Israel, South Africa and Broome, Australia identified as Phascolosoma (P.) nigrescens correspond to different species. It is very likely that other species from those regions, considered synonyms, would need to be reinstated following redescription, or described and established as new species. 2) It will be necessary to resolve a potential species complex of Phascolosoma $(P$.) nigrescens by combining molecular and morphological data. Neither one of these considerations were resolved in the present study, as they were beyond the original scope of this investigation.

Peer) reviewing PDF | (2020:08:51760:1:0:NEW 22 Sep 2020) 
441

442

443

444

445

446

447

448

449

450

451

452

453

454

455

456

457

458

459

460

461

462

463

464

465

466

467

468

469

470

471

472

\section{Conclusions \\ Conclusions}

474

475

476

477

478

479

480 coloration patterns. varians is reinstated.

The assumed wide distribution of Sipuncula taxa is attributed to the development of the teleplanic larvae in some species, such as Phascolosoma (Phascolosoma) varians. Although it is well accepted that the free-swimming larval stage with its prominent locomotive organ (known as a metatroch) confers the ability to disperse, allowing an increase in geographic range and providing for genetic exchange between populations (Rice, 1981), it is not necessarily the rule. Staton \& Rice (1999) described the case of a species with teleplanic larvae with a limited distribution. They found distinct genetic differences within and between larval and adult stages of Apionsoma (A.) misakianum (Ikeda, 1904) from northern and southern regions of Florida and the Bahamas using allozymes. They did not find any indication of "hybrids" occurring between them, suggesting a potential oceanic boundary was present between populations with teleplanic pelagosphera larvae, and thus a possible Apionsoma species-complex in the region. They did not perform a morphological analysis of the adults at that time, but the larve exhibited two distinct

Kawauchi \& Giribet (2010) rejected the cosmopolitanism of Phascolosoma (P.) perlucens Baird, 1868, by analyzing molecular and morphological data of specimens from many localities around the world. They detected four different lineages, and identified variation in hook morphologies between different localities that correlated with a high genetic diversity between populations. Also, their results suggested a probable lack of gene flow between the geographically distinct lineages. Schulze et al. (2012) analyzed molecular sequence data and developmental features of three "cosmopolitan" species, Phascolosoma (P.) agassizii Keferstein, 1866, Thysanocardia nigra (Ikeda, 1904), and Themiste (T.) pyroides (Chamberlin, 1920). For each one of the three species, they found significant differences between previously assumed con-specific populations from the Sea of Japan and the Northeast Pacific region, with respect to egg size, developmental mode and developmental timing. The populations of all three species were remarkably distinct genetically and suggested that gene flow between the two regions was extremely unlikely. Furthermore, Kawauchi \& Giribet (2014) analyzed the genetic data from four genes of Sipunculus (S.) nudus Linnaeus, 1766 with specimens from multiple localities worldwide. As with Phascolosoma perlucens (see above), these two investigators again found high levels of genetic differentiation between distantly related populations, suggesting in this case there were five distinct lineages, three of which could be distinguished morphologically. In the last two studies, neither a new species was described, nor an available name reinstated.

High genetic divergence between specimens identified as $P$. P. nigrescens from the Greater Caribbean and a region close to its type locality correlate with morphological differences found between $P$. $(P$.$) varians and P$. $(P$.$) nigrescens. Herein, the synonymy of Phascolosoma (P$. varians with Phascolosoma $(P$.$) nigrescens is rejected, and as a consequence, Phascolosoma (P$.

Based upon these findings, and other studies, some of which are discussed here, the diversity of sipunculans is most likely underestimated. Thus, a combination of morphological 
481

482

483

484

485

486

487

488

489

490

491

492

493

494

495

496

497

498

499

500

501

502

503

504

505

506

507

508

509

510

511

512 Frontana-Uribe SC, Hermoso-Salazar M, Solís-Weiss V. 2018. Sipunculans from intertidal and 513

and molecular data, along with other important information from the fields of development, ecology and oceanography, will help us determine a more realistic number of extant sipunculans species worldwide.

\section{Acknowledgements}

I would like to thank Luis F. Carrera-Parra (ECOSUR) who contributed greatly with his recommendations and comments to improve the manuscript, and his production of SEM photos. Thanks to Nancy Voss (UMML) and Gustav Paulay (UF) who kindly lent the specimens used in this study. Thanks to Sergio I. Salazar-Vallejo (ECOSUR) and Luis F. Carrera-Parra who provided specimens from the Collection of Benthos of El Colegio de la Frontera Sur, Chetumal, Quintana Roo, Mexico. Thanks to Gerardo Flores-Taboada for his comments and for reviewing the translation. Thanks to Mario H. Londoño-Mesa and to an anonymous reviewer for their suggestions to improve this manuscript.

\section{References}

Boyle MJ, Rice ME. 2014. Sipuncula: an emerging model of spiralian development and evolution. The International journal of developmental biology, 58: 485-499. DOI: https://doi.org/10.1387/ijdb.140095mb

Cutler EB. 1994. The Sipuncula. Their systematics, biology and evolution. Ithaca, N.Y.: Cornell University Press.

Cutler EB, Cutler NJ. 1983. An examination of the Phascolosoma subgenera Antillesoma, Rueppellisoma, and Satonus (Sipuncula). Zoological Journal of the Linnean Society $77: 175-187$.

Cutler NJ, Cutler EB. 1990. A revision of the subgenus Phascolosoma (Sipuncula, Phascolosoma). Proceedings of the Biological Society of Washington, 103: 691-730.

Cutler EB, Schulze A. 2004. Sipuncula from Barbados, including two new for the island plus Siphonosoma vastum; first record from the Atlantic Ocean. Bulletin of Marine Science, 74(1): 225-228.

De Quatrefages MA. 1865. Annélides et géphyriens. Paris: Histoire Naturelle des Annelés Marins et d'Eau Douce.

lower subtidal coralline substrates of the Mexican Caribbean Sea. In: Boyle MJ, 
514

515

516

517

518

519

520

521

522

523

524

525

526

527

528

529

530

531

532

533

534

535

536

537

538

539

540

541

542

543

Kawauchi GY, eds. Proceedings of the Second International Symposium on the Biology of the Sipuncula. Washington, D.C.: Smithsonian Institution Scholarly Press, 169-185.

Hebert PDN, Ratnasingham S, de Waard JR. 2003. Barcoding animal life: cytochrome c oxidase subunit 1 divergences among closely related species. Proceedings of the Royal Society of London Series B-Biological Sciences 270: S96-S99.

HeliconSoft Limited. 2007. HeliconFocus 4.21 (blend the focused areas). Available at http://www.heliconsoft.com (accesed 05 March 2020).

Hylleberg J. 2013. Classification and identification of Sipunculans from Thailand, with description of new species and a new subgenus. Phuket Marine Biological Center, Research Bulletin, 32: 53-82.

Johnson ND, Sanders C, Maiorova A, Schulze A. 2016. Cryptic species in Pacific sipunculans (Sipuncula: Phascolosomatidae): east-west divergence between non-sister taxa. Zoologica Scripta, 45(4):455-463. DOI: https://doi.org/10.1111/zsc.12158

Kawauchi GY, Giribet G. 2010. Are there true cosmopolitan sipunculan worms? A genetic variation study within Phascolosoma perlucens (Sipuncula, Phascolosomatidae). Marine Biology, 157(7):1417-1431. DOI http://dx.doi.org/10.1007/s00227-010-1402-z

Kawauchi GY, Giribet G. 2014. Sipunculus nudus Linnaeus, 1766 (Sipuncula): cosmopolitan or a group of pseudo-cryptic species? An integrated molecular and morphological approach. Marine Ecology, 35(4):478-491. DOI: https://doi.org/10.1111/maec.12104

Kawauchi GY, Rice ME. 2009. Two new species of Nephasoma (Sipuncula: Golfingiidae) from the western Atlantic Ocean. Proceedings of the Biological Society of Washington, 122(1): 1-13. DOI: http://dx.doi.org/10.2988/08-32.1

Kawauchi GY, Sharma PP, Giribet G. 2012. Sipunculan phylogeny based on six genes, with a new classification and the descriptions of two new families. Zoologica Scripta, 41(2):186-210. DOI: http://dx.doi.org/10.1111/j.1463-6409.2011.00507.x

Keferstein W. 1865. Beiträge zur anatomischen und systematischen Kenntniss der Sipunculiden. Zeitschrift für Wissenschaftliche Zoologie, 15: 404-445.

Kimura M. 1980. A simple method for estimating evolutionary rates of base substitutions through comparative studies of nucleotide sequences. Journal of Molecular Evolution, 16(2): 111-120 DOI: http://dx.doi.org/10.1007/BF01731581 
544 Kumar S, Stecher G, Tamura K. 2016. MEGA7: molecular evolutionary genetics analysis

545

546

547

548

549

550

551

552

553

554

555

556

557

558

559

560

561

562

563

564

565

566

567

568

569

570

571

572

573 version 7.0 for bigger datasets. Molecular Biology and Evolution, 33(7):1870-1874. DOI: http://dx.doi.org/10.1093/molbev/msw054

Quiroz-Ruiz MM, Londoño-Mesa MH. 2015. Sipunculans (Sipuncula) from the Great Caribbean: Species list and bibliography. Bulletin of Marine and Coastal Research, 44(2):327-342.

Rice ME. 1970. Observations of the Development of Six Species of Caribbean Sipuncula with a Review of Development in the Phylum. In Proceedings of the International Symposium on the Biology of the Sipuncula and Echiura. Kotor.

Rice ME. 1976. Larval development and metamorphosis in Sipuncula. American Zoologist, 16:563-571. DOI: https://doi.org/10.1093/icb/16.3.563

Rice ME. 1981. Larvae adrift: patterns and problems in life histories of sipunculans. American Zoologist, 21(3) 605-619. DOI: https://doi.org/10.2307/3882614

Rice ME, Macintyre I.G. 1982. Distribution of Sipuncula in the Coral Reef Community, Carrie Bow Cay, Belize. In: The Atlantic Barrier Reef Ecosystem at Carrie Bow Cay, Belize, I STRUCTURE AND COMMUNITIES, Eds. Riitzler K. \& Macintyre I.G. Smithsonian contributions to the Marine Sciences, 12: 311-320

Saiz JI, Bustamante M, Tajadura J, Vijapure T, Sukumaran S. 2015. A new subspecies of Phascolion Théel, 1875 (Sipuncula: Golfingiidae) from Indian waters. Zootaxa, 3931(3): 433-437. DOI: https://doi.org/10.11646/zootaxa.3931.3.7

Selenka E. 1883. Die Sipunculiden, eine systematische Monographic, Reisen in Archipel Phillippinen von Dr. C. Semper. Wiesbaden.

Scheltema RS, Rice ME. 1990. Occurrence of teleplanic pelagosphera larvae of sipunculans in tropical regions of the Pacific and Indian Oceans. Bulletin of Marine Science, 47(1): 159181.

Schulze A. 2005. Sipuncula (Peanut Worms) from Bocas del Toro, Panama. Caribbean Journal of Science, 42(3): 523-527.

Schulze A, Maiorova A, Timm LE, Rice ME. 2012. Sipunculan larvae and "cosmopolitan" species. Integrative and Comparative Biology, 52(4):497-510. DOI: http://dx.doi.org/10.1093/icb/ics082 
574 Schulze A, Rice ME. 2004. Sipunculan diversity at Twin cays, Belize with a key to the species.

575

576

577

578

579

580

581

582

583

584

585

586

587

588

589

590

591

592

593

594

595 Atoll Research Bulletin, 1-9. DOI: 10.5479/si.00775630.521.1-9

Shipley AE. 1890. On Phymosoma varians. Quarterly Journal of Microscopical Science, 31: 127.

Silva-Morales I, López-Aquino MJ, Islas-Villanueva V, Bastida-Zavala JR, Ruiz-Escobar F. 2019. Morphological and molecular differences between the amphiamerican populations of Antillesoma (Stephen \& Edmonds, 1972) (Sipuncula: Antillesomatidae), with the description of a new species. Revista de Biología Tropical, 67(S5):101-109. DOI: https://doi.org/10.15517/RBT.V67IS5.38934

Staton J, Rice ME. 1999. Genetic differentiation despite teleplanic larval dispersal: Allozyme variation in sipunculans of the Apionsoma misakianum species complex. Bulletin of Marine Science, 65(2):467-480.

Stephen AC, Edmonds SJ. 1972. The phyla Sipuncula and Echiura. London: Trustees of the British Museum (Natural History)

Tamura K. 1992. Estimation of the number of nucleotide substitutions when there are strong transition-transversion and $\mathrm{G}+\mathrm{C}-$ content biases. Molecular Biology and Evolution, 9(4): 678-687. DOI: https://doi.org/10.1093/oxfordjournals.molbev.a040752

ten Broeke JMA. 1925. Westindische Sipunculiden und Echiuriden. Bijdragen tot de Dierkunde, 24(1): 81-96. DOI: https://doi.org/10.1163/26660644-02401006

Wesenberg-Lund E. 1954. Priapuloidea, Sipunculoidea and Echiuridea. Bulletin de l'Institut royal des Sciences naturelles de Belgique, Biologie, 30(16): 1-18. 


\section{Figure 1}

Papillar morphology on Phascolosoma (Phascolosoma) varians Keferstein, 1865

Regional variation in the patterns of papillae along the body are shown for specimen UF 332, from Saint Martin, West Indies. (A) Adult body plan in dorsal view with anterior to the top. (B) Papillae from introvert region with light transversal bands. (C) Papillae from introvert. (D) Papillae from anal region of trunk. (E) Papillae from median region of trunk. (F) Papillae from caudal region of trunk. Scale bars = A, $2 \mathrm{~mm}$; B-C, $0.3 \mathrm{~mm}$; D, $1 \mathrm{~mm}$; E-F, $0.5 \mathrm{~mm}$. White arrow indicates the location of the anus. 


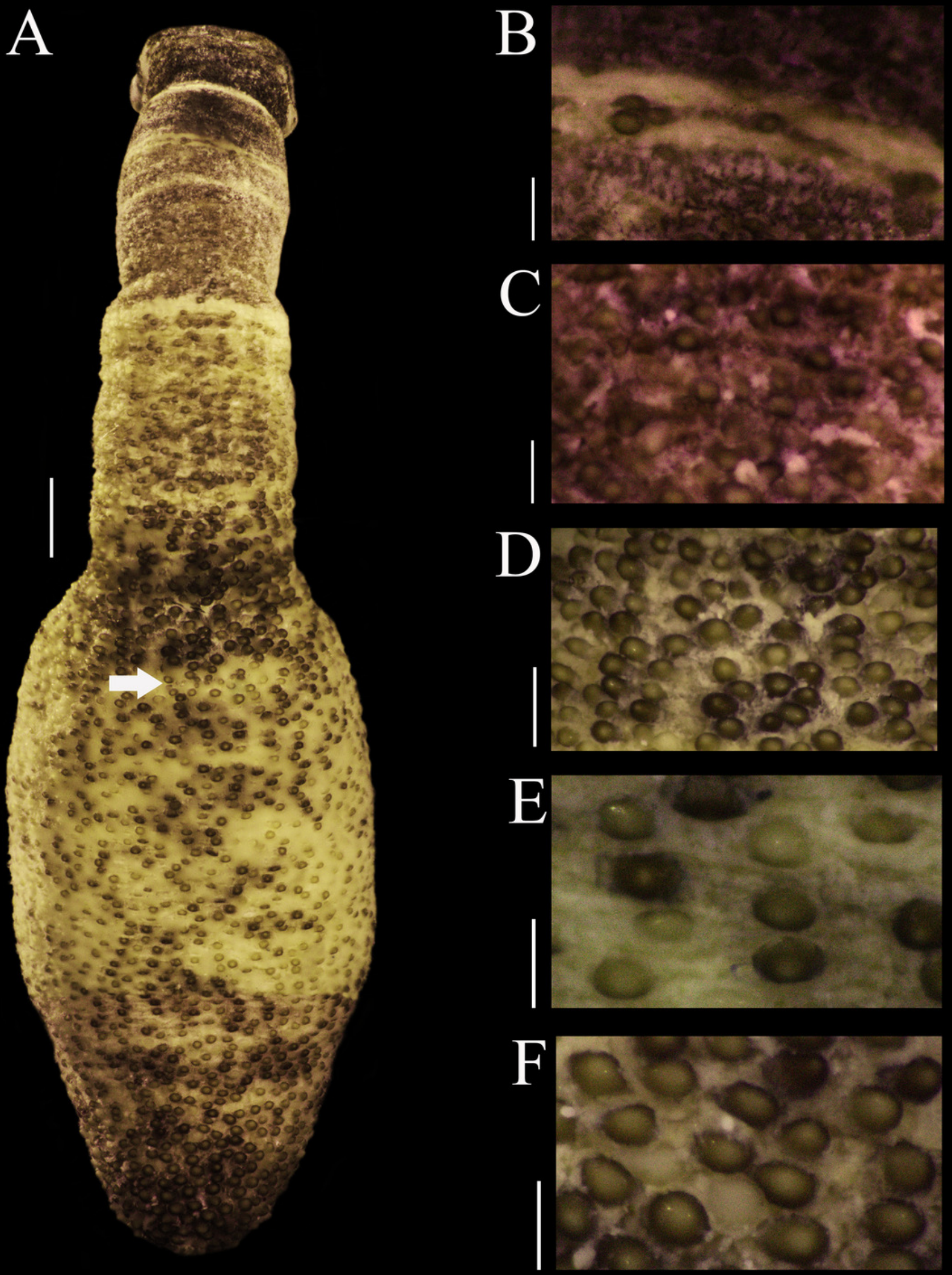




\section{Figure 2}

Anatomical characters of Phascolosoma (Phascolosoma) varians Keferstein, 1865

Micrographs of hook, muscle and papillar anatomy are shown for specimen UF 332, from

Saint Martin, West Indies, unless stated. (A) Proximal hooks, (B) hooks of median region of introvert and (C) distal hooks. (D) Internal anatomy of a dissected specimen, with anterior to the top. (E) Posterior attachment site of a ventral retractor muscles (VRM) associated with longitudinal muscle bands 1-9; specimen ECOSUR-S94 from the Mexican Caribbean. (F) SEM image of hook from anterior region of the introvert; specimen UF359 from St. Martin, West Indies. (G) SEM image of hooks from median region of the introvert. (H) SEM image showing stages of hook growth; from left right: first seven rings of introvert hooks, arrows show individual papillae between rows of hooks. (I) SEM image of papilla from distal introvert. (J) SEM image of papilla from median region of the introvert; specimen UF 26.5 from Florida. (K) SEM image of papilla from proximal introvert. Abbreviations: DRM, dorsal retractor muscles; IN, intestine; LMB, longitudinal muscle bands; N, nephridia; SM, spindle muscle; SEM, scanning electron microscope; VRM, ventral retractor muscles. Scale bars $=A, B, C, 30 \mu \mathrm{m}$; F, G, I, J, $20 \mu \mathrm{m} ; \mathrm{H}, 50 \mu \mathrm{m} ; \mathrm{K}, 5 \mu \mathrm{m}$. 


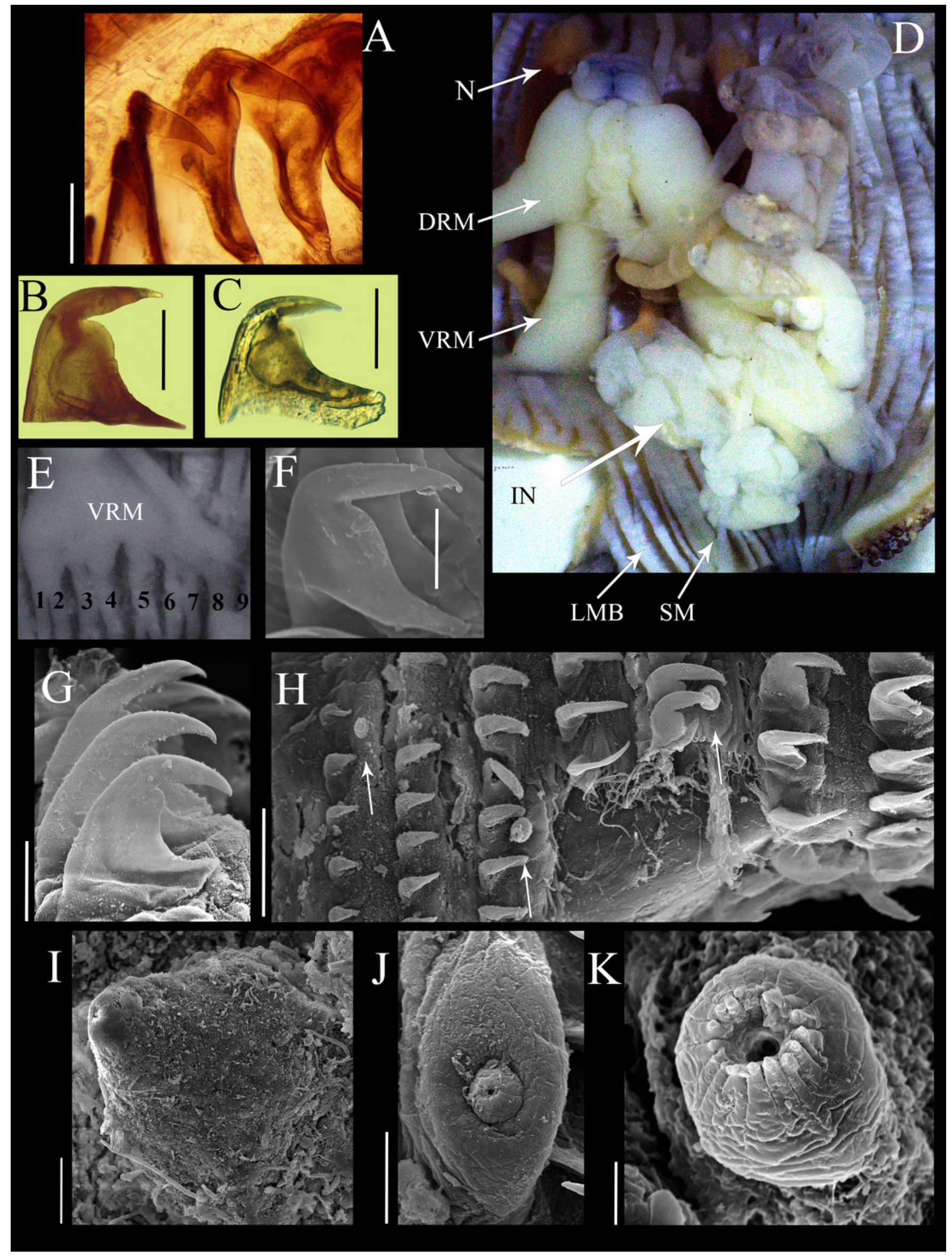




\section{Figure 3}

Hooks and papillae of Phascolosoma varians and Phascolosoma nigrescens.

From drawings of (A-D) Phymosoma varians, Barbados and (E-H) Phymosoma nigrescens, Fiji Islands, by Selenka (1883). (A) Trunk hook. (B) Papilla between the hook rings on introvert. (C) Papilla from side in longitudinal section. (D) Skin of the middle of the body with domed papillae. (E) Trunk hook. (F) Middle of papilla in ' $G$ ' from above. (G) Papilla between the scattered introvert hooks. (H) Domed body papilla at 140X magnification. Both species originally described by Keferstein, 1865. 

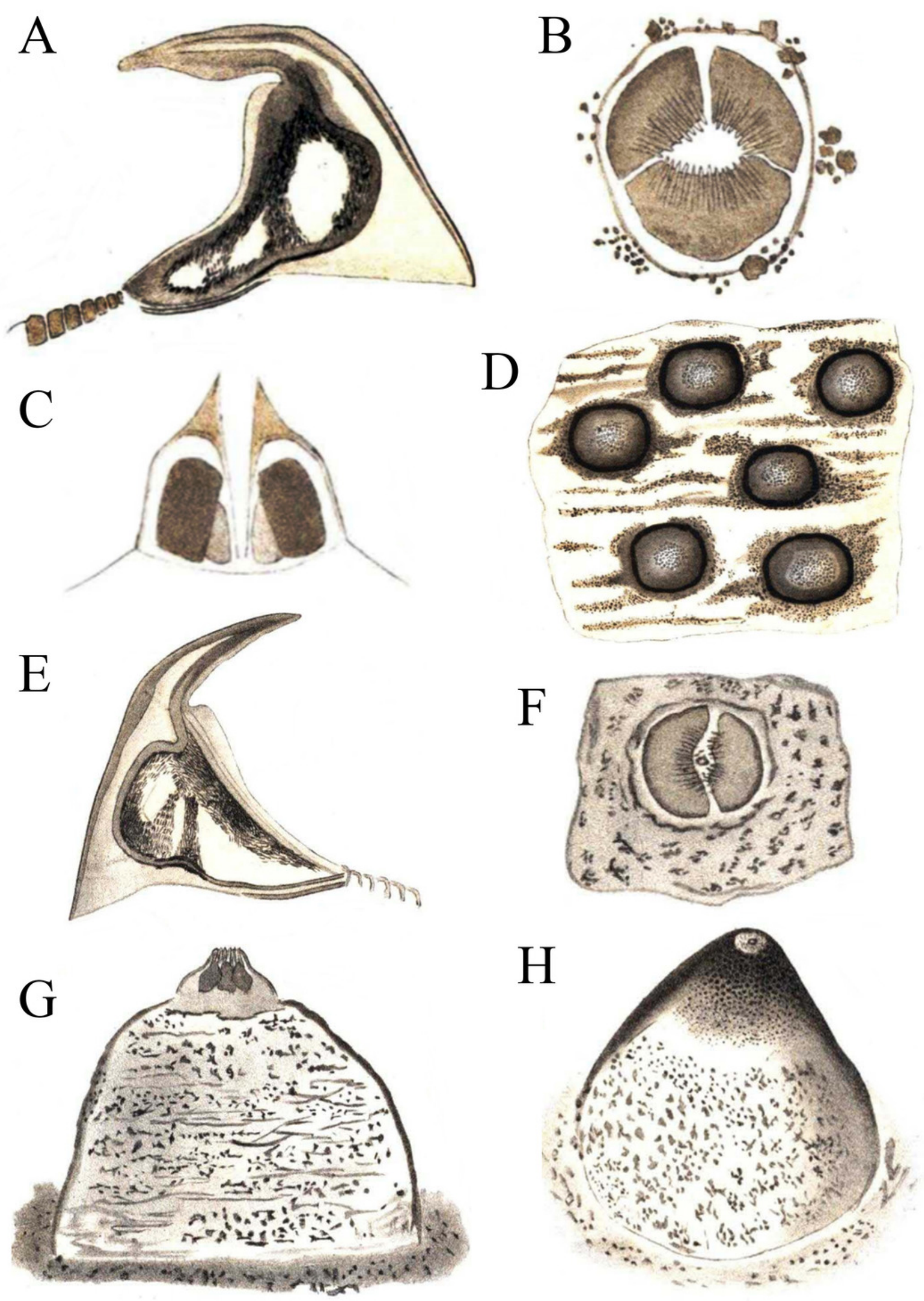


\section{Figure 4}

Maximum likelihood analysis of cytochrome c oxidase subunit 1 (COI) DNA.

Phascolosoma (P.) varians from the Greater Caribbean shows a clear genetic distinction from all specimens identified as Phascolosoma ( $P$.) nigrescens from eastern and western regions of the Pacific Ocean. Tree reconstruction was generated from a ClustalW alignment of COI sequences amplified from 11 sipunculan specimens. Species names (specimens) and GenBank accession numbers are listed at the branch tips. Individuals and conspecific groups are marked with vertical bars, and their corresponding geographic regions, to the right of species names. This analysis was performed using Tamura 3-parameter with a discrete Gamma distribution with five rate categories, assuming a certain fraction of sites is evolutionarily invariable $(\mathrm{T} 92+\mathrm{G}+\mathrm{I})$. 


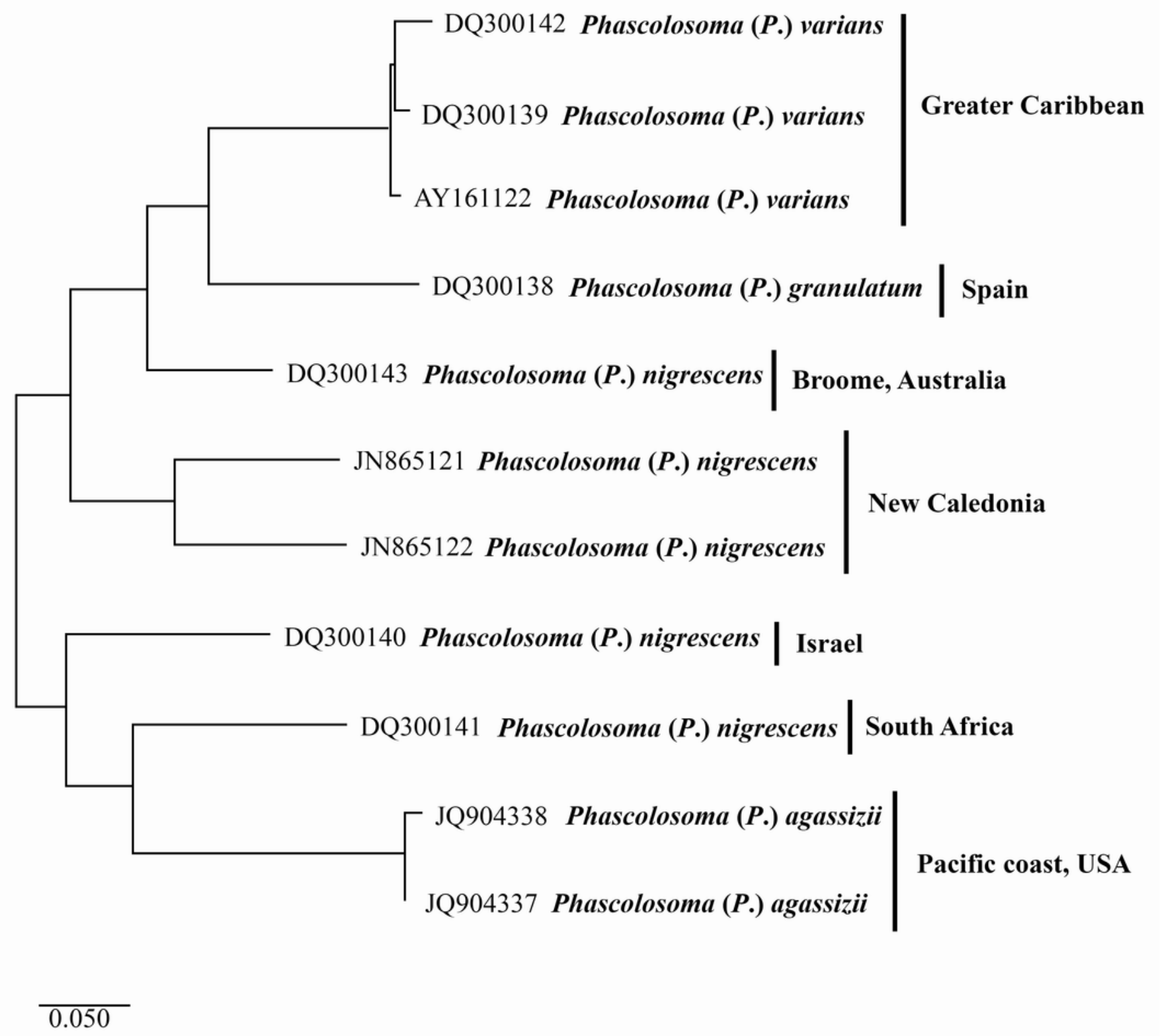

